\title{
Bidimensional Empirical Mode Decomposition Modified for Texture Analysis
}

\author{
J.C. Nunes, O. Niang, Y. Bouaoune, E. Delechelle, and Ph. Bunel \\ LERISS \\ Laboratoire d'Etude et de Recherche \\ en Instrumentation, Signaux et Systèmes \\ Université Paris XII-Val de Marne \\ 94010 CRETEIL Cedex - FRANCE \\ nunes@univ-paris12.fr
}

\begin{abstract}
This study introduces a new approach based on Bidimensional Empirical Mode Decomposition (BEMD) to extract texture features at multiple scales or spatial frequencies. Moreover, it can resolve the intrawave frequency modulation provided the frequency modulation. This decomposition, obtained by the bidimensional sifting process, plays an important role in the characterization of regions in textured images. The sifting process is realized using morphological operators to analyze the spatial frequencies and thanks to radial basis functions (RBF) for surface interpolation. We modified the original sifting algorithm to permit a pseudo bandpass decomposition of images by inserting scale criterion. Its effectiveness is demonstrated on synthetic and natural textures. In particular, we show that many different elements in textures can be extracted through the bidimensional empirical mode decomposition, which is fully unsupervised.
\end{abstract}

\section{Introduction}

Four major texture analysis categories may be identified [1]: statistical [2], geometrical [3], model based [4] [5] and multiresolution methods. The multiresolution filtering decompositions, which are widely used in computer vision, are inspired by visual texture perception. The most commonly multiscale methods used are morphological decomposition [6], spectrogram [7], AM-FM analysis [8], Wigner-Ville Distributions [9], Gabor functions [10] and wavelets transforms [11]. These multiresolution techniques intend to transform images into a representation in which both space and spatial frequency information are present.

Several authors made a comparison of the performance of various operators and features for texture segmentation, but they have different points of sights on the most suitable method [12]. Moreover, no single approach did perform best or very close to the best for all images.

We present a pseudo bandpass decomposition of texture images, based on Bidimensional Empirical Mode Decomposition (BEMD), firstly introduced in [21]. This multiresolution procedure, proposed by Hang in 1D [13], is such that each spatial frequency band of the image is obtained by EMD, according to a predefined criterion (neighboring distance). This scale criterion corresponds to spatial frequency bands of 
the original texture. We also show the adaptability of the bandwidth decomposition, by applying it to a variety of textures.

The paper is organized as follow: in section II, we present a first version of the BEMD. A modified BEMD, which integrate a neighboring distance criterion, is given in section III. In section IV, texture analysis is described and properties of their results are examined. In section $\mathrm{V}$, we summarize the results of the study and draw conclusions.

\section{Texture analysis based on the BEMD}

The EMD approach has been recently introduced in signal processing by Huang in [13]. This decomposition technique is adaptive and appears to be a suitable for non linear, non-stationary data analysis. We applied the EMD on image analysis. The Bidimensional EMD, introduced in [21, 22] permits to extract zero-mean 2D AM-FM components called Intrinsic Mode Functions (IMF).

\subsection{The Bidimensional Empirical Mode Decomposition}

This decomposition, derived from image data, is developed from the simple assumption that any data consist of different simple intrinsic modes of oscillations. Its principle is to decompose adaptively a given signal into AM-FM components or IMF. Each IMF has the same number of zero crossings and extrema (minus one) and each IMF is symmetric with respect to the a zero mean. For each mode, the algorithm, which extracts locally the highest frequency oscillations out of original signal, is called "sifting process".

\subsubsection{Bidimensional sifting process}

Bidimensional sifting process is defined as follow [21]:

- $\quad$ Detect the extrema (both maxima and minima) of the image $I$ by morphological reconstruction ( $\mathrm{hmin} / \mathrm{hmax}$ );

- Compute the 2-D upper and lower envelope by connecting extrema points with radial basis functions;

- $\quad$ Determine the mean $m$ of upper and lower envelopes;

- $\quad$ Subtract out the mean from the image: $\quad I-m=h$

- $\quad$ Repeat until $h$ is an IMF.

The EMD repeats bidimensional sifting process, after subtracting of original image by precedent IMF, until there is no more IMF, i.e. not enough extrema. It only remains the image residue. Components superposition reconstructs the data:

$$
I=\sum_{i=1}^{n}\left(c_{i}\right)+r_{n}
$$

To stop the IMF extraction, we used the standard deviation (SD), computed from the two consecutive sifting results as:

$$
S D=\sum_{l=0}^{L} \sum_{k=0}^{K}\left[\frac{\left|\left(h_{i-1}(l, k)-h_{i}(l, k)\right)\right|^{2}}{h_{i}^{2}(l, k)}\right]
$$

In practice, we have used SD between 0.02 and 0.3 and this stop criteria gives satisfying results. 


\subsubsection{Regional Extrema detection}

The morphological reconstruction is applied to find all extrema (minima and maxima) during the sifting process. Vincent et al [15] [16] proposed geodesic operators (hmin/hmax), which permit to extract "domes" or "valleys" of a definite size. For more details of this step see [22].

\subsubsection{Surface interpolation by radial basis functions}

In [13], Huang proposed to use cubic spline interpolation on non-equidistant sampled data. We have choice to use the radial basis function (RBF) rather than the bicubic spline for different reasons developed in [17], as regularization properties. RBF constitutes a powerful tool for working with 2D data non-uniformly sampled [18].

\subsection{The modified BEMD}

In $1 \mathrm{D}$, the sifting process may run into difficulties when the data contains intermittency, which will cause mode mixing. Although, these mixed modes can have very different frequencies, it causes intermittency occurring in part of the 1D signal. To overcome the mode mixing, Huang [19] proposed a criterion based on the period length.

For image analysis, we also propose to introduce a distance or a scale criterion. This discrimination enables to obtain modes comprising of the areas having texture elements according to a well defined distance between extrema.

\subsubsection{Algorithm of Modified BEMD}

The modified sifting algorithm for $v \in l^{2}\left(\mathbb{Z}^{2}\right)$, where $v$ is the original image, reads as follows:

1) Initialise: $r_{0}=v$ (the residual) and $j=1$ (index number of IMF),

2) Extract the $j^{\text {th }}$ IMF:
a. Initialise $h_{0}=r_{j-1}, i=1$.
b. Extract regional extrema (minima/maxima) of $h_{i-1}$.
c. Search the minimal spatial frequency from extrema.
i. Compute the distance images from extrema.
ii. Compute the watershed of distance images
iii. Select the extrema which have neighbours having neighbour- ing lower than $\mathrm{k}$ times the minimal neighbouring surface, where $k$ is a frequency scale factor.

d. Compute upper envelope and lower envelope functions $x_{i-1}$ and $y_{i-1}$ by interpolating respectively local minima and local maxima of $h_{i-1}$, those which check the criterion of the minimal distance.

e. Compute $m_{i-1}=\left(x_{i-1}+y_{i-1}\right) / 2$.

f. In region surrounding where distance criteria don't be verified, place the original image values in mean envelope. 
g. $\quad$ Update $h_{i}:=h_{j-1}-m_{i-1}$, and $i:=i+1$.

h. Compute stopping criterion SD (Standard Deviation).

i. Repeat step (b) to (g) until $S D_{i} \leq \varepsilon$ and put then $v_{j}=h_{i}\left(j^{\text {th }} I M F\right)$.

3) Update residual $r_{i j}=r_{j-1}-v_{j}$,

4) Repeat step 1-3 with $j:=j+1$ until the number of extrema in $r_{j}$ is less than two.

We compute the Euclidean distance transform of the binary extrema image figure 1(b). For each pixel in extrema image, the distance image assigns a number that is the distance between this pixel and the nearest nonzero pixel of extrema image. To calculate the lines peaks of the distance image, we use the watershed morphological operator.

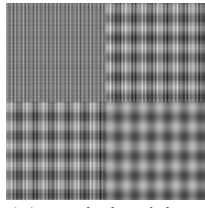

(a): original image (b): minima

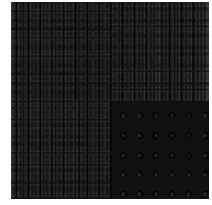

Fig. 1. We present a synthetic image, regional minima, the distance images between extrema., the watershed computed from distance images and a mask image obtained from scale criteria.

The mask image enables to compute the mean of the upper and lower envelopes. On the extrema that check the criterion, we apply the sifting process. On the others extrema, we assign to the average image the values of the residual image. This distance criterion makes possible the detection of areas corresponding to a definite frequential band. Thus, our decomposition introduce a stage of segmentation.

\subsubsection{The choice of scale factor}

In $1 \mathrm{D}$, the period length is introduced to separate the waves of different periods into different modes. For image analysis, we propose to introduce a distance or a scale criterion. Initially, the value of $k$ can be given in an empirical way. From the image of the extrema, we build the histogram of the points distributions in frequency bandwidth. By statistical measurements, a scale factor is estimated in each mode.

\section{Results and discussion}

We chose the EMD, as their good discrimination properties of scale and spatial frequencies are well-known in 1D. We applied BEMD at textured images. Our approach gives a simultaneous representation of an image in space and spatial frequency representation. It is an unsupervised texture decomposition. 


\subsection{Texture extraction}

Since sifting process extracts firstly the highest frequency, the first modes correspond generally to the noise. Conversely, the image tendency is contained in the latest mode. The tendency can be represented by a polynomial of order relatively low $(0,1$, 2, 3).

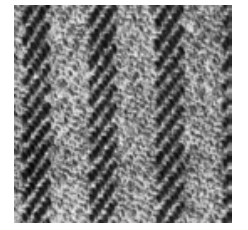

(a): original image

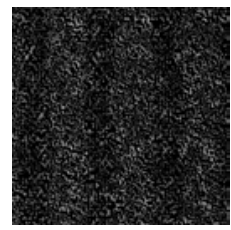

(b): mode 1

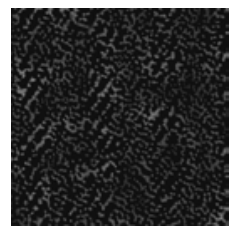

(c): mode 2

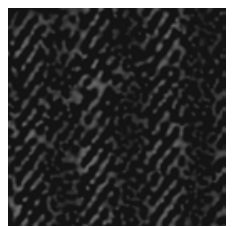

(d): mode 3

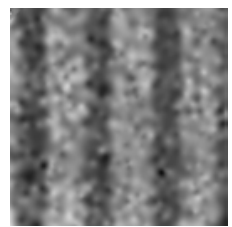

(e): residue image

Fig. 2. Brodatz image is decomposed by BEMD in 3 modes and a residue image.

Figure 2(a) shows the D11 brodatz texture [20]. The BEMD is shown in figure 2(b, $\mathrm{c}, \mathrm{d}$ and e) and is clearly useful for discriminating the texture features: broad band vertical and fine oblique bands.

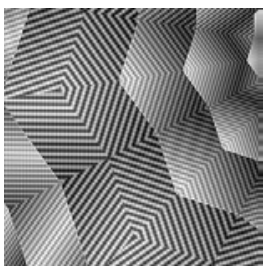

(a): original image

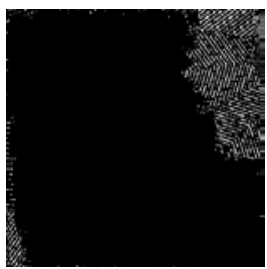

(b): mode 1

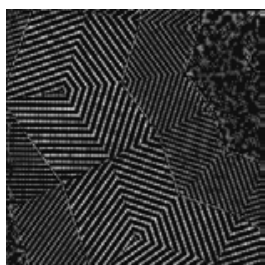

(c): mode 2

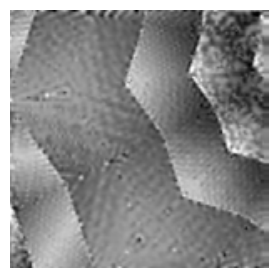

(d): residue image

Fig. 3. Synthetic image is decomposed by BEMD in 2 modes and a residue image.

Figure 3(a) shows a synthetic image with a juxtaposition of textured regions, which have different spatial frequencies and orientations. The results of BEMD are shown in figure $3(\mathrm{~b}, \mathrm{c}$ and $\mathrm{d})$. We chose a scale criteria $k$ equal to 10 . The first IMF corresponds to the areas of image where the local frequency is included in the bandwidth of minimal frequency and 10 times minimal frequency.

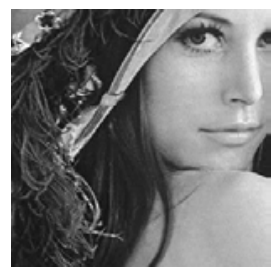

(a): original image

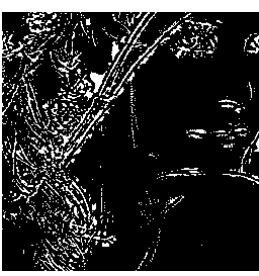

(b): mode 1

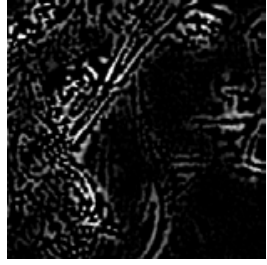

(c): mode 2

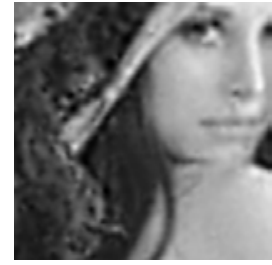

(d): residue image

Fig. 4. Experimental results of Lena image is decomposed by BEMD in 2 modes and a residue image.

We apply modified BEMD on Lena image Figure 4(a) decomposed in 2 modes and the residue Figure 4(b, c, d). $\mathrm{k}$ is equal to 80 . This decomposition allows a unsupervised texture segmentation. The edges are extracted in the first modes. 


\subsection{Discussion}

A benefit of bidimensional Empirical Mode Decomposition is their high conjoint resolution, which means that their response (mode) is highly localized in both space and spatial frequency and is adaptive with respect to the global information . In 1D, Huang proposed a pseudo bandpass to extract intermittencies. Scale criterion can be computed automatically and rather than given in an empirical way. During the sifting process, we statistically study the size of the areas of neighboring defined by watershed lines of distance images. The modified EMD are also suitable for texture segmentation. We estimate a criterion of scale but we can also estimate a shape criterion corresponding to the areas of neighboring. The shape criterion could be geometrical characteristics as compactness, eccentricity, direction, ...

\section{Conclusion}

We developed an algorithm based on BEMD, introuduced in [21] for texture extraction, and demonstrate its effectiveness on synthetic and natural textures. It provides a powerful characterization of the local texture structure. We have modified the bidimensional sifting process to permit a pseudo-bandpass decomposition by inserting a scale criteria. By combining EMD with scale criteria, we extract texture features. This could be computed during the sifting process from the neighbouring distance and shape between extrema. This texture analysis method is fully unsupervised and includes a segmentation process.

\section{References}

[1] M. Tuceryan and A. K. Jain, "Texture analysis", The Handbook of pattern Recognition and Computer Vision (2nd edition), by C. H. Chen, L. F. Pau, P. S. P. Wang (editors.), 207-248, World scientific Publishing Co., 1998.

[2] R. Haralick, "Statistical and structural approaches to texture", IEEE Proc., 67(5): 1979, 786-804.

[3] M. Tuceryan and A. K. Jain, "Texture Segmentation Using Voronoi Polygons," IEEE Trans. on Pattern Analysis and Machine Intelligence, vol. PAMI-12, pp. 211216, February, 1990.

[4] C.C. Chen, J.S. Daponte, and M.D. Fox, "Fractal feature analysis and classification in medical imaging", IEEE Transactions on Medical Imaging , 8, 133-142, 1989.

[5] B. S. Manjunath and R. Chellappa, "Unsupervised texture segmentation using Markov radom field models", IEEE. Trans. Pattern Anal. Machine Intell., 13(5):478482, May 1991.

[6] R. A. Peters II, "Morphological pseudo bandpass image decompositions", Journal of Electronic Imaging, vol. 5, n², April 1996, 198-213.

[7] J. Krumm and S.A. Shafer, "Shape from Periodic Texture Using Spectrogram," Proc. IEEE Conf. Computer Vision and Pattern Recognition, pp. 284-289, 1992.

[8] J.P. Havlicek, D.S. Harding, and A.C. Bovik, "Multidimensional quasieigenfunction approximations and multicomponent AM-FM models", IEEE Trans. Image Proc., vol. 9, no. 2, pp. 227-242, February 2000. 
[9] J. Hormigo and G. Cristóbal, "High Resolution Spectral Analysis of Images Using the Pseudo-Wigner Distribution", IEEE Transactions on Signal Processing, vol. 46, no. 6, 1757-1763, June 1998.

[10] D. Dunn and W. E. Higgins, "Optimal Gabor filters for texture segmentation", IEEE Trans. Image Proc., 4(7):947-964, july 1995

[11] M. Unser, "Texture classification and segmentation using wavelet frames", IEEE Trans. Image Proc., 4(11):1549-1560, November 1995.

[12] T. Randen J.H. Husoy, "Filtering for texture classification: a comparative study", IEEE Trans. Patt. Anal. Machine Intell., 21:291-310, 1999.

[13] N. E Huang and al., "The empirical mode decomposition and the Hilbert spectrum for non-linear and non-stationary time series analysis", Proceedings of the Royal Society Lond. A, 454, 903-995, 1998.

[14] P.J. Oonincx; "Empirical mode decomposition: a new tool for S-wave detection", CWI Reports of Probability, Networks and Algorithms (PNA) 2002, PNAR0203, ISSN 1386-3711

[15] L. Vincent, "Morphological grayscale reconstruction in image analysis: applications and efficient algorithms", IEEE Transactions on Image Processing, Vol. 2, No. 2, 176-201, April 1993.

[16] P. Soille, "Morphological Image Analysis: principles and applications", Springer Verlag, 1999, 170-171.

[17] J.C. Carr, W.R. Fright, and R.K. Beatson, "Surface interpolation with radial basis functions for medical imaging", IEEE Trans. Med. Imag. vol. 16, pp. 96-107, janv. 1997.

[18] T. Blu, and M. Unser, "Wavelet, fractals and radial basis functions", IEEE Trans. on Signal Processing. vol. 50 (3), pp. 543-553, Mar. 2002.

[19] N. E Huang, Z. Shen and S. R. Long, "A new view of nonlinear water waves: the Hilbert spectrum”, Annu. Rev. Fluid. Mech., 1999, 31: 417-57.

[20] P. Brodatz, "Textures: a photographic album for artists and designers", New York Dover publications, 1966.

[21] J.C. Nunes, Y. Bouaoune, E. Deléchelle, S. Guyot, and Ph. Bunel. "Texture analysis based on the bidimensional empirical mode decomposition". Journal of Machine Vision and Applications, (to appear), 2003.

[22] J.C.Nunes, Y. Bouaoune, E. Deléchelle, O. Niang, and Ph. Bunel. "Image analysis by Bidimensional Empirical Mode Decomposition". Image and Vision Computing Journal , (to appear), 2003. 